\title{
Excitons, biexcitons, and phonons in ultrathin CdSe/ZnSe quantum structures
}

Gindele, F.; Woggon, Ulrike; Langbein, Wolfgang Werner; Hvam, Jørn Märcher; Leonhardi, K.; Hommel, D.; Selke, H.

Published in:

Physical Review B

Link to article, DOI:

10.1103/PhysRevB.60.8773

Publication date:

1999

Document Version

Publisher's PDF, also known as Version of record

Link back to DTU Orbit

Citation (APA):

Gindele, F., Woggon, U., Langbein, W. W., Hvam, J. M., Leonhardi, K., Hommel, D., \& Selke, H. (1999).

Excitons, biexcitons, and phonons in ultrathin CdSe/ZnSe quantum structures. Physical Review B, 60(12), 87738782. https://doi.org/10.1103/PhysRevB.60.8773

\section{General rights}

Copyright and moral rights for the publications made accessible in the public portal are retained by the authors and/or other copyright owners and it is a condition of accessing publications that users recognise and abide by the legal requirements associated with these rights.

- Users may download and print one copy of any publication from the public portal for the purpose of private study or research.

- You may not further distribute the material or use it for any profit-making activity or commercial gain

- You may freely distribute the URL identifying the publication in the public portal 


\title{
Excitons, biexcitons, and phonons in ultrathin CdSe/ZnSe quantum structures
}

\author{
F. Gindele and U. Woggon \\ Institut für Experimentelle Physik, Universität Dortmund, D-44221 Dortmund, Germany \\ W. Langbein and J. M. Hvam \\ Mikroelektronik Centret, Technical University of Denmark, DK-2800 Lyngby, Denmark \\ K. Leonardi and D. Hommel \\ Institut für Festkörperphysik, Universität Bremen, 28359 Bremen, Germany
}

H. Selke

Institut für Werkstoffphysik und Strukturforschung, Universität Bremen, D-28359 Bremen, Germany

(Received 28 October 1998)

\begin{abstract}
The optical properties of CdSe nanostructures grown by migration-enhanced epitaxy of CdSe on $\mathrm{ZnSe}$ are studied by time-, energy-, and temperature-dependent photoluminescence and excitation spectroscopy, as well as by polarization-dependent four-wave mixing and two-photon absorption experiments. The nanostructures consist of a coherently strained $\mathrm{Zn}_{1-x} \mathrm{Cd}_{x} \mathrm{Se} / \mathrm{ZnSe}$ quantum well with embedded islands of higher $\mathrm{Cd}$ content with sizes of a few nanometer due to strain-induced CdSe accumulation. The local increase in CdSe concentration results in a strong localization of the excitonic wave function, in an increase in radiative lifetime, and a decrease of the dephasing rate. Local LO-phonon modes caused by the strong modulation of the Cd concentration profile are found in phonon-assisted relaxation processes. Confined biexcitons with large binding energies between 20 and $24 \mathrm{meV}$ are observed, indicating the important role of biexcitons even at room temperature. [S0163-1829(99)16935-2]
\end{abstract}

\section{INTRODUCTION}

The current activities to realize quantum dots in epitaxially grown II-VI semiconductors are based both on the interest in fundamental aspects of three-dimensional-quantum confinement and the search for new optoelectronic materials. Further lowering of the dimensionality from quantum wells toward quantum dots is very promising to achieve laser emission in the visible to deep blue spectral range. Epitaxial growth of three-dimensionally confined II-VI materials has recently attracted intensive research, ${ }^{1-8}$ and a variety of growth methods are investigated. For example, StranskiKrastanow growth, ${ }^{5}$ thermally activated self-assembling after growth of a few monolayers, ${ }^{9}$ or submonolayer growth ${ }^{6,10}$ have been reported to initiate the formation of quantum dots. As has been demonstrated in Ref.11, islands of enhanced CdSe concentrations can be obtained within a $\mathrm{Zn}_{1-x} \mathrm{Cd}_{x} \mathrm{Se}$ quantum well of varying composition, when using the method of migration-enhanced epitaxy (MEE).

For the confined excitonic states forming in these structures, different quantum dot models are proposed in the literature, e.g. quantum confinement in pure CdSe islands or the formation of three-dimensional excitons in local potentials due to well width fluctuations. Quantum dots are introduced as the result of strain-induced local potentials in $\mathrm{Zn}_{1-x} \mathrm{Cd}_{x}$ Se quantum wells in Ref. 12 .

Presently, a quantitative investigation of the $\mathrm{CdSe} / \mathrm{ZnSe}$ structure formed after the overgrowth with a ZnSe cap layer is rather difficult. Segregation and diffusion processes during the $\mathrm{ZnSe}$ capping procedure give rise to distinct differences between the uncapped and final structures. Also, the nature and origin of islands observed at uncapped structures by ex situ atomic force microscopy is still controversially discussed $^{2,11,13,14}$.

In this paper, we propose a combined quantum-wellquantum-dot model for the MEE-grown CdSe/ZnSe quantum structures under study. We start with a description of the growth process, and present results of structural analysis by high-resolution transmission electron microscopy (HRTEM). Based on these data we put forward the structural model, and in Sec. III calculate the exciton transition energies as they depend on composition and well thickness. The results are compared with experimental data obtained by photoluminescence (PL) and excitation spectroscopy (PLE). In Sec. IV, we discuss the phonon-assisted relaxation processes in these structures. Different types of phonons are detected in micro-PL and energy-selective PLE, showing energies smaller than the CdSe zone center LO-phonon and in the range between the $\mathrm{CdSe}$ and $\mathrm{ZnSe} \mathrm{LO}$ phonons. The energy distribution of the LO phonons involved in the exciton relaxation process monitors the local density of phonon states according to the $\mathrm{Cd}$ distribution within the spatially inhomogeneous quantum structure. The dynamics of the exciton localization and the decay of the coherence are studied in Secs. $\mathrm{V}$ and VI. Changing the detection energy, the transition from extended to localized excitonic wave functions can be followed in the change of radiative lifetimes and dephasing times. In the final section (Sec. VII), we study the formation and decay of biexcitons using time-resolved PL after twophoton absorption and femtosecond four-wave mixing (FWM). A strong enhancement of the biexciton binding energy compared to bulk CdSe as a result of the threedimensional quantum confinement is found. 

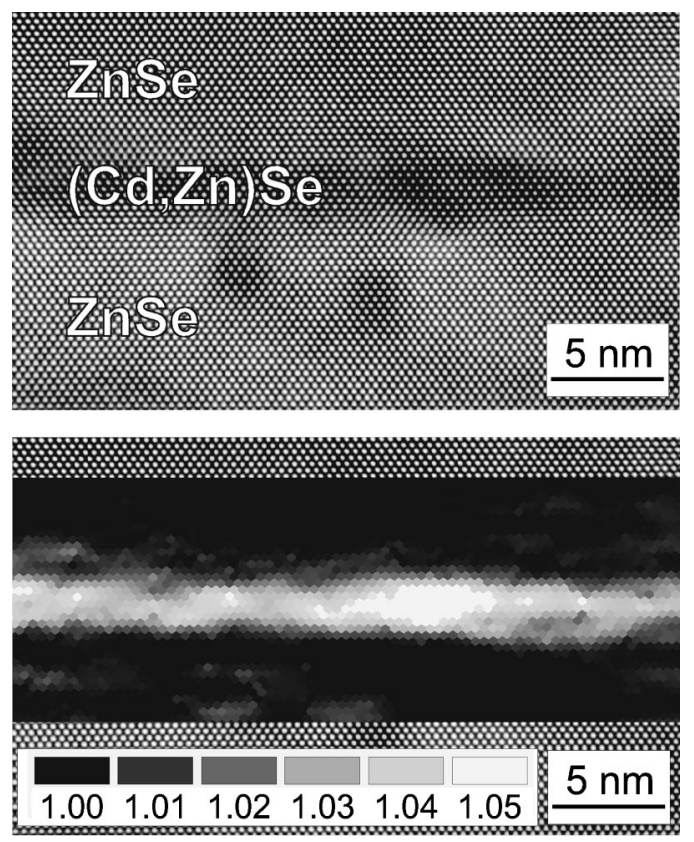

FIG. 1. (a) HRTEM image of a single CdSe quantum film (arrows) embedded in $\mathrm{ZnSe}$ of nominal (deposited) layer thickness of $3 \mathrm{ML}$, and (b) result of digital analysis of local lattice displacement (DALI). The gradient in the gray scale shows the increase in lattice constants starting from the pure ZnSe lattice (black) up to approximately $50 \% \mathrm{CdSe}$ (white).

\section{SAMPLE CHARACTERIZATION}

The samples under study have been grown by molecularbeam epitaxy (MBE) on GaAs(001) substrates in a twinchamber MBE system. A GaAs buffer layer is deposited prior to the growth of the II-VI heterostructure. The thin CdSe quantum films are grown between a 40-nm ZnSe barrier layer and a $20-\mathrm{nm} \mathrm{ZnSe}$ cap layer. As surface diffusion is known to play an important role for the island formation in heteroepitaxy, MEE has been applied. For the growth of $\mathrm{CdSe}$ on $\mathrm{ZnSe}$, typical growth rates of 0.3-0.5 ML per MEE cycle have been used at a substrate temperature of $280^{\circ} \mathrm{C}$. In order to increase surface migration of ad-atoms during the growth a delay has been introduced between consecutive depositions of $\mathrm{Cd}$ and Se. ${ }^{7,11}$ The nominally deposited CdSe thickness derived from the number of MEE cycles has been varied between 1 and $8 \mathrm{ML}$. The transition from two- to three-dimensional growth is monitored in situ by the change of the reflection high-energy electron diffraction from a streaky pattern to a spotty pattern. ${ }^{11}$ Diffusion processes during the deposition of the final $\mathrm{ZnSe}$ cap layer, however, significantly modify the morphology of the grown $\mathrm{CdSe}$, since interdiffusion of $\mathrm{Cd}$ in $\mathrm{ZnSe}$ is particularly strong. ${ }^{15-17} \mathrm{To}$ extract the actual distribution of $\mathrm{Cd}$ in the overgrown structure, high-resolution cross-sectional transmission electron microscopy combined with a digital analysis of the lattice images (DALI) ${ }^{18,19}$ has been applied. CdSe is found to grow in a cubic modification during the deposition of the first few monolayers. Figure 1(a) shows a HRTEM image of a sample with a deposited (nominal) CdSe thickness of 3 ML. Fluctuations in the $\mathrm{Cd}$ distribution are visible in the intensity contrast between $\mathrm{Cd}$ and $\mathrm{Zn}$ in the upper image. The analysis of the local lattice constants by means of DALI gives direct information about the local $\mathrm{Cd}$ concentration as displayed in
Fig. 1(b). The gradient in the gray scale shows the increase in lattice constants starting from the pure ZnSe lattice (black) and ending at about 50\% Cd (white). Because of the finite sample thickness of a few tens of nanometers used in the HRTEM, the data represent an average over many lattice constants in depth, and the local Cd concentration is expected to fluctuate even stronger. Figure 1 shows that the investigated structure consists of a $\mathrm{Zn}_{1-x} \mathrm{Cd}_{x} \mathrm{Se}$ quantum film of about 9-ML thickness with $x \geqslant 0.3$. The quantum film exhibits only small fluctuations of the layer thickness, but a strong variation of the $\mathrm{Cd}$ concentration. Islands with $\mathrm{Cd}$ concentrations larger than $50 \%$ are found. These islands are not towering above the well, as observed, e.g., for InAs grown on GaAs. ${ }^{20,21}$ The lateral island size is about $5-10$ $\mathrm{nm}$, i.e. of the order of the bulk exciton Bohr radius in CdSe $\left(a_{B}=5.6 \mathrm{~nm}\right)$. From the analysis of HRTEM images, we conclude that the investigated $\mathrm{CdSe} / \mathrm{ZnSe}$ quantum structures consist of $\mathrm{Zn}_{1-x} \mathrm{Cd}_{x} \mathrm{Se} / \mathrm{ZnSe}$ quantum wells with islands of increased $\mathrm{Cd}$ concentration inside. This structure presumably evolves from the initially formed layer/island structure during the overgrowth with the $\mathrm{ZnSe}$ cap layer as the result of $\mathrm{Cd}$ diffusion.

\section{WELL-DOT MODEL}

The results from the structural analysis of Sec. II allow us to model the electronic states in the samples as a combination of a flat, coherently strained $\mathrm{Zn}_{1-x} \mathrm{Cd}_{x} \mathrm{Se} / \mathrm{ZnSe}$ quantum well with quantum dots of higher $\mathrm{Cd}$ concentration inside. We first calculate the energies of the heavy-hole (hh) and light-hole (lh) states of the undisturbed homogeneous quantum well in dependence on the composition $x$ and the well width $L$. Starting from the effective-mass model for zincblende-type semiconductors, finite potential barriers and strain effects have been included using the Hamiltonian of Bir and Pikus. ${ }^{22}$ To take into account the Coulomb potential, the transition energies have been corrected by the twodimensionally-confined exciton energy of $2 E_{\mathrm{exc}}^{\text {bulk }}$, a value which is reasonable for finite potential barriers ( $E_{\mathrm{exc}}^{\text {bulk }}$ $=15 \mathrm{meV}$ is the CdSe bulk exciton binding energy). Due to the strong lattice mismatch between $\mathrm{CdSe}$ and $\mathrm{ZnSe}$ of $6.8 \%$, biaxial compression in the $\mathrm{Zn}_{1-x} \mathrm{Cd}_{x} \mathrm{Se}$ layer shifts the valence and conduction bands. The $\mathrm{ZnSe}$ buffer is nearly lattice matched to GaAs $(0.3 \%)$, and grows coherently over the full structure.

The elastic deformation can be expressed as a superposition of hydrostatic and shear strain, giving rise to the hydrostatic deformation potential shift $E_{\text {hyd }}$, which effects both conduction and valence bands, and the shear deformation potential shift $E_{\mathrm{sh}}$, which only affects the valence band. Therefore, in addition to the quantum confinement effect, hh and $\mathrm{lh}$ states are split by the deformation potential. The strain-induced energy difference between the conduction and valence bands at $\vec{k}=0$ is given, in first order, by ${ }^{22}$

$$
\begin{gathered}
\Delta E_{\mathrm{hh}}=E_{\mathrm{hyd}}-E_{\mathrm{sh}}, \\
\Delta E_{\mathrm{lh}}=E_{\mathrm{hyd}}+\frac{1}{2}\left(E_{\mathrm{sh}}-\Delta_{\mathrm{SO}}+\sqrt{\Delta_{\mathrm{SO}}^{2}+2 \Delta_{\mathrm{SO}} E_{\mathrm{sh}}+9 E_{\mathrm{sh}}^{2}}\right),
\end{gathered}
$$

with 

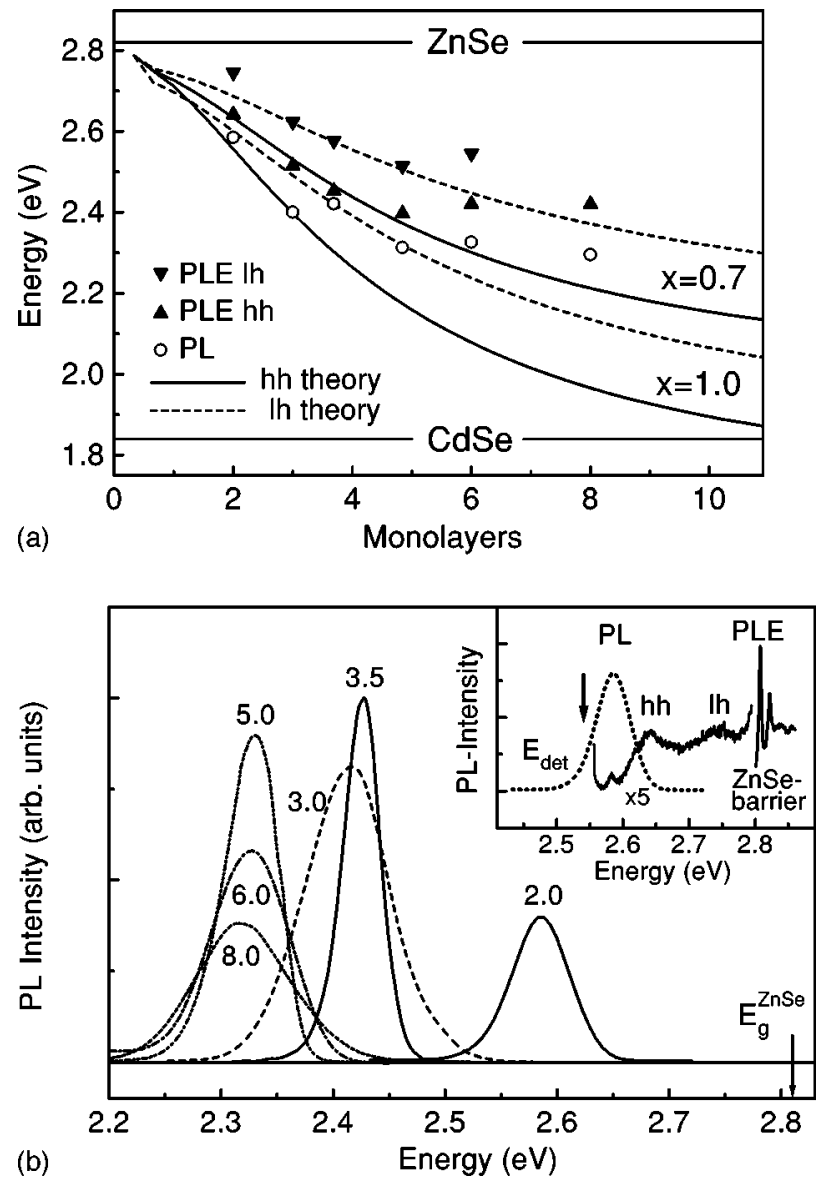

FIG. 2. (a) Calculated transition energies of the hh and lh states for a strained, two-dimensional $\mathrm{Zn}_{1-x} \mathrm{Cd}_{x} \mathrm{Se} / \mathrm{ZnSe}$ structure with $x$ $=1$ and 0.7 as a function of well width. The experimental data obtained by PLE (triangles) and PL (circles) are plotted for a set of samples with a well thickness between 2 and 8 ML measured by HRTEM and x-ray scattering. (b) PL spectra for the same set of samples. The inset shows a typical PLE spectrum for the 2-ML sample detected in the tail of the PL.

$$
\begin{gathered}
E_{\mathrm{hyd}}=2 a e_{0} \frac{C_{11}-C_{12}}{C_{11}}, \\
E_{\mathrm{sh}}=b e_{0} \frac{C_{11}+2 C_{12}}{C_{11}} ; \quad e_{0}=\frac{a_{\mathrm{ZnSe}}-a_{\mathrm{CdSe}}}{a_{\mathrm{CdSe}}} .
\end{gathered}
$$

$a$ and $b$ are the hydrostatic and shear deformation potentials, respectively, $e_{0}$ is the lattice mismatch, $C_{i i}$ the elastic moduli, and $\Delta_{\text {SO }}$ the spin-orbit splitting constant. For $z \|(001)$ the components of the in-plane strain tensor are given by $\epsilon_{x x}=\epsilon_{y y}=\epsilon_{0}$. For the alloy, values of the deformation potentials, the elastic constants, the effective masses, etc., can be obtained by linear interpolation between the pure binary materials, ${ }^{23}$ keeping in mind that for the used materials some parameters are not known with good accuracy, as, e.g., the deformation potentials. We use here the values of $a=a_{\mathrm{c}}-a_{\mathrm{v}}=-3.664$ and $-4.25 \mathrm{eV}$, and $b=-0.8$ and $-1.2 \mathrm{eV}$ for the deformation potentials of CdSe and $\mathrm{ZnSe}$, respectively, according to Ref. 24. The composition dependence of the band gap is taken from Ref. 25. The band offsets of the unstrained bulk materials are $230 \mathrm{meV}$ for the valence band and $750 \mathrm{meV}$ for the conduction band.
Figure 2(a) shows the energies of the hh and lh states as a function of well width $L$ calculated for an ideal twodimensional $\mathrm{Zn}_{1-x} \mathrm{Cd}_{x} \mathrm{Se} / \mathrm{ZnSe}$ quantum structure with $x$ $=1$ and 0.7 . With increasing $\mathrm{Zn}$ concentration, the transition energies shift to higher values. To compare the calculation with the confined energy states of the structures under study, PL and PLE have been applied. An overview of the PL spectra of the different samples is given in Fig. 2(b). The average thickness of the quantum well which surrounds the islands has been taken from HRTEM/DALI analysis, as illustrated in Fig. 1(b). The energies of the deepest confined states which give rise to the PL signal are influenced by the well thickness $L$ and the composition $x$. As shown in Sec. II, fluctuations are predominantly caused by changes in the composition within the well, while the well thickness itself is rather constant. Therefore, the lowest states from which the PL signal will arise form in the islands of enhanced CdSe content. The absorption process and thereby the PLE signal, however, is mainly expected at photon energies corresponding to more extended states from which the excitons can relax and emit at the detection energy. The PLE signal thus mainly comprises information about the composition of the surrounding quantum well, having a larger density of states, whereas the PL signal is dominated by the properties of the islands, having smaller transition energies.

The inset of Fig. 2(b) shows a PLE spectrum for a 2.0-ML sample when detecting in the low-energy tail of the PL band. The spectral shape of the PLE (besides one phonon replica, seen as a small peak at $2.59 \mathrm{eV}$ ) is constant for detection energies within the low-energy PL wing. Therefore, contributions from excited states of the islands and due to relaxation processes between different islands do not significantly modify the PLE spectra at higher energies. The two broadbands above the detection energy are attributed to the inhomogeneously broadened hh and lh exciton states of the surrounding $\mathrm{Zn}_{1-x} \mathrm{Cd}_{x} \mathrm{Se} / \mathrm{ZnSe}$ quantum well. By analyzing the PLE spectra of the samples studied, the energies of these transitions can be determined as a function of (average) well width. In Fig. 2(a), the experimentally determined energies of the hh and $\mathrm{lh}$ exciton states (triangles) are compared with the calculated curves. The experimentally obtained energies are in disagreement with the calculation for a pure CdSe well $(x=1)$. Better agreement is found for $x=0.7$ or even lower $\mathrm{Cd}$ content.

The energy shift between PL [circles in Fig. 2(a)] and PLE (triangles), the so-called Stokes shift, is a measure of the strength of composition fluctuations. This Stokes shift varies between the different samples. The 3.5-ML sample is an exception in structure and linewidth. In HRTEM it shows a nearly flat quantum well of $\mathrm{Zn}_{0.3} \mathrm{Cd}_{0.7}$ Se with only small thickness fluctuations. The PL spectrum is characterized by the narrowest linewidth, and the smallest Stokes shift of 32 $\mathrm{meV}$. The majority of the MEE-grown samples, however, exhibit pronounced composition fluctuations in HRTEM, which are reflected in the optical properties by large Stokes shifts of up to $140 \mathrm{meV}$.

With increasing well thickness, the PL maximum converges to a nearly constant emission energy, as can be seen in Fig. 2. We explain this behavior by a quenching of the luminescence due to dislocations which form during strain relaxation and act as nonradiative recombination centers. 


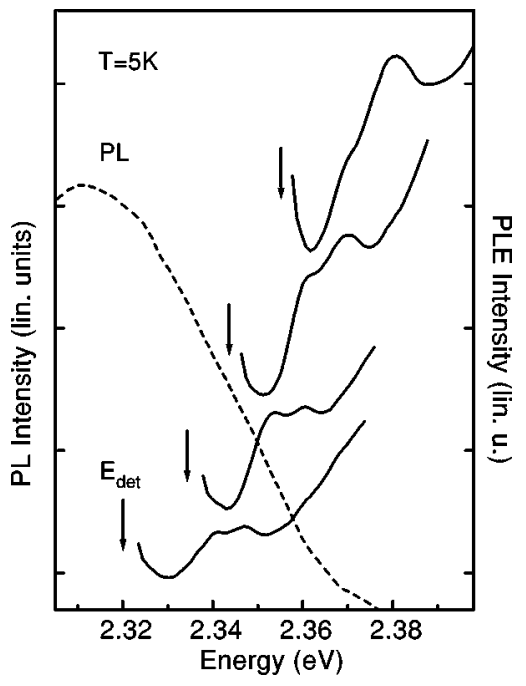

FIG. 3. Phonon double structure observed in PLE for a 5-ML $\mathrm{Zn}_{1-x} \mathrm{Cd}_{x} \mathrm{Se} / \mathrm{ZnSe}$ structure measured for different detection energies. The PL is shown as the dashed curve.

Since the islands with a large $\mathrm{Cd}$ accumulation have the largest probability to form defects, the Cd content in optically active dots converges to an upper limit, and the shift of the PL maximum saturates with an increasing amount of deposited $\mathrm{CdSe}$ together with a reduction of the overall PL efficiency.

\section{LOCAL EXCITON-PHONON INTERACTION}

A peculiarity of the grown quantum structures is the variation of their local composition on a nanometer scale. The polar II-VI semiconductors $\mathrm{ZnSe}$ and CdSe show a strong exciton-optical-phonon coupling, giving rise to strong LO-phonon replica in the optical properties (see, e.g., Refs. 26 and 27). Because of the strongly modulated $\mathrm{Cd}$ concentration in the samples we expect spatially different LO-phonon frequencies. By investigation of phonon-assisted processes, we can thus probe the energy distribution and density of state of local phonon modes which couple to the localized excitons. Figures 3 and 4 show results from energy-selective PLE and microphotoluminescence ( $\mu$-PL) experiments. It was shown in Ref. 28 that PLE spectra of systems with an inhomogeneous broadening larger than the LO-phonon energy depend sensitively on the detection energy within the PL band. Detecting on the high-energy side of the PL, the PLE shows a series of equidistant peaks with an energy difference close to the LO-phonon energy of the barrier material. At these energies, a further relaxation into deeper localized states via coupling to LO phonons is faster than relaxation via acoustic phonons into these states ${ }^{29}$ and the PLE spectra are dominated by LO-phonon relaxation.

To study the LO-phonon coupling of the excitons localized in the islands, PLE experiments were performed at various detection energies $E_{\text {det }}$. The data are plotted in Fig. 3 for the 5-ML sample (see Fig. 2), which shows a large Stokes shift of $84 \mathrm{meV}$. A double peak can be identified in the PLE signal about one LO-phonon energy above the detection energy. The two peaks clearly have their origin in the $\mathrm{ZnSe}$ and CdSe LO phonons but their energy separation and relative intensities are changing when tuning $E_{\text {det }}$. With increasing

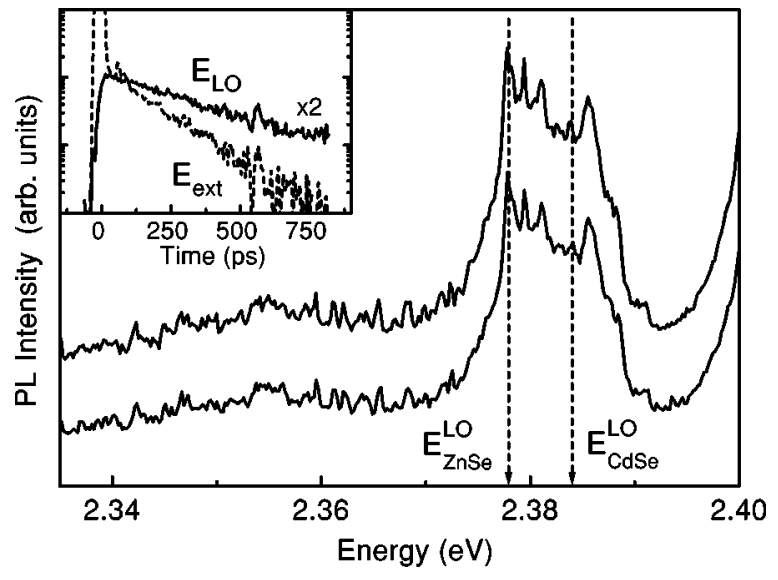

FIG. 4. Microphotoluminescence spectra excited at $514 \mathrm{~nm}$ in the tail of the density of states of the 3-ML sample. The energy resolution is $\leqslant 0.3 \mathrm{meV}$. The decay curves detected at the excitation energy $E_{\text {ext }}$ and at the first LO-phonon replica $E_{\mathrm{LO}}$ are shown in the inset.

detection energy the phonon energy of the higher-energy peak decreases from 31.5 to $27 \mathrm{meV}$, and increases in intensity relative to the low-energy peak, that also shows a shift to smaller energies. We attribute the change in the phonon energies to mainly three mechanisms: (i) formation of mixed LO-phonon modes with energies between the pure $\mathrm{ZnSe}$ and CdSe zone-center LO-phonons (as shown in Refs. 24 and 30 the mixed crystal $\mathrm{Zn}_{1-x} \mathrm{Cd}_{x} \mathrm{Se}$ shows one-mode behavior, and the LO-phonon energy changes monotonically with composition $x$ ); (ii) strain-induced changes of the LOphonon frequencies and, in particular, for structures thinner than $2 \mathrm{~nm}$; (iii) confinement of the phonon modes which results in a reduction of the phonon energy due to the decrease in LO-phonon energy away from the quasimomentum zone center. This is especially important for the CdSe-like phonon modes, which are localized in the CdSe islands, and partly show energies even smaller than the bulk zone-center LO-phonon energy of $26 \mathrm{meV}$. With increasing detection energy, the $\mathrm{Cd}$ content of the local environment decreases, which is evidenced by the observed change in the intensity ratio favoring the higher energetic $\mathrm{Zn}_{1-x} \mathrm{Cd}_{x} \mathrm{Se}$-well LO phonon. This is in agreement with the behavior expected from the electronic band structure proposed in Sec. III.

When probing localized excitons with higher spatial resolution, we can resolve individual LO-phonon assisted transitions. In Fig. 4 we show the PL excited with the 514-nm line of the $\mathrm{Ar}^{+}$laser resonantly in the low-energy tail of the density of excitonic states, and detected with a spatial resolution of $1.5 \mu \mathrm{m}$. A low excitation intensity has been chosen, and by measuring of the intensity dependence of the PL signal it has been ensured that the linear regime holds for all PL peaks. Thus effects of biexciton formation (see Sec. VII) are negligible in that experiment. The broad first LO-phonon replica shows a substructure of several narrow peaks with energies in the same energy range as the doublet structure observed in PLE. As can clearly be seen, LO-phonon energies below the CdSe bulk LO phonon are also involved in the phonon assisted transitions. The inset of Fig. 4 shows the dynamics of the PL signal measured at the excitation energy $E_{\text {ext }}$, and the energy of the first LO-phonon replica $E_{\mathrm{LO}}$ (de- 


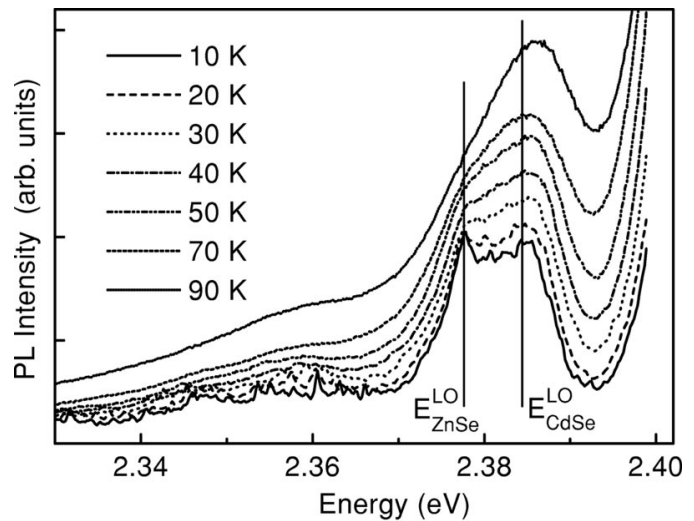

FIG. 5. Temperature dependence of the first LO-phonon replica in microphotoluminescence at the same conditions as in Fig. 4.

tected with a spatially wide excitation; details are given in Sec. V). The PL decay times are clearly different, indicating that the LO-phonon replica is due to a population of real exciton states at this energy, and not due to phonon-assisted recombination over virtual states. Nonresonant Raman scattering can be excluded, since no instantaneous contribution is observed at the LO-phonon energy. Also, phonon-assisted absorption is unlikely to explain the replica because of the strongly decreasing density of states with decreasing energy. Sharp, reproducible peaks in the PL are also present in the energy range of the second LO-phonon replica about $50 \mathrm{meV}$ below the excitation, as can be seen from the comparison of two subsequent measurements in Fig. 4 excluding random noise. A part of the confined excitons thus show an energy relaxation emitting local LO phonons of different energy, and finally populate deeper localized states. The experimental result of Fig. 4 shows that narrow peaks in $\mu$-PL appear as the result of phonon-assisted relaxation between localized states.

The temperature dependence of the phonon-assisted relaxation is shown in Fig. 5. With increasing temperature, the sharp features disappear, and the coupling to the LO phonons with smaller energies (e.g., $\mathrm{CdSe}$ ) is more weighted by the Bose-Einstein statistical factor in the scattering rate for phonons. Additionally, some localized excitons become thermally activated and redistributed in the potential landscape by multiple-phonon scattering, forming a broad unstructured PL band.

\section{DYNAMICS OF LOCALIZED EXCITONS}

To study the dynamics of exciton localization, most of the experiments ${ }^{31-33}$ have used an excitation energy resonant to the band of delocalized excitons, and the subsequent luminescence decay was analyzed by rate equations of a multilevel system. Such an approach always results in a manyparameter model. The relaxation process proceeds via subsequent population of spatially distributed local exciton states, and excitons are considered as particles moving around in a potential landscape. The driving force for the relaxation is a spatial variation of the potential depth. During its lifetime the exciton relaxes to local-energy minima accompanied by the emission of acoustic and optical phonons. The two time constants which characterize the dynamics are
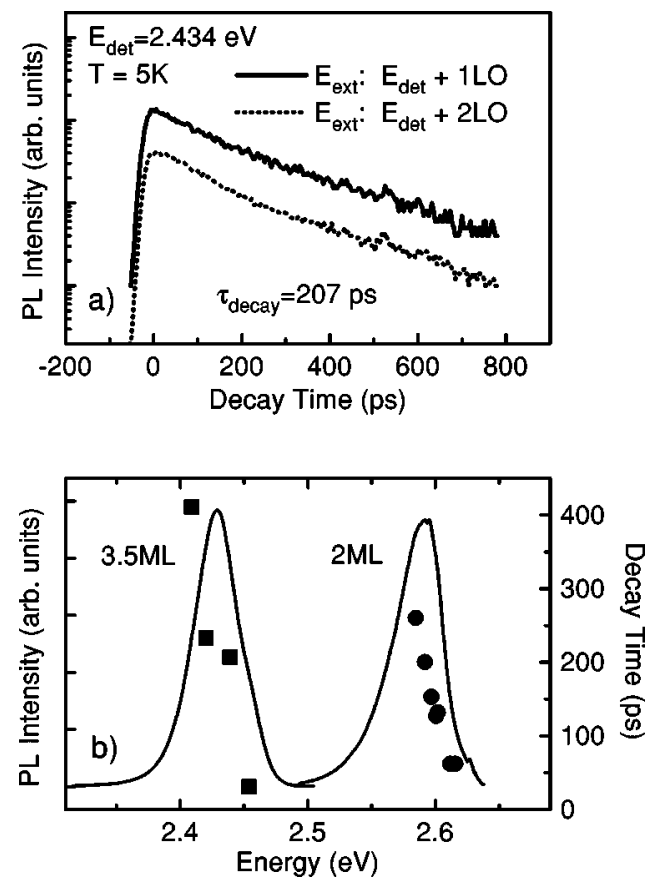

FIG. 6. (a) PL decay measured for the 3.5-ML sample when exciting one- and two LO-phonon energies above the detection energy. (b) PL decay times plotted as a function of the detection energy for the 3.5-ML sample and the 2-ML sample. For comparison, the nonresonantly excited PL spectra are shown.

the lifetime of the exciton (radiative and nonradiative recombination) and the migration time between local potential minima. ${ }^{32}$

For the experiments presented here, we start from the idea that quasiresonant excitation of localized excitons considerably reduces the number of free parameters in the kinetics of exciton relaxation. By performing an experiment with only small excess energies, we expect that migration between the localized states can be neglected. The PL decay should be almost monoexponential, and predominantly defined by the lifetime of the localized excitons itself. The exciton dynamics are studied by excitation with 2-ps pulses of a frequencydoubled mode-locked Ti-sapphire laser and detection by a streak camera, providing a temporal resolution of 4 ps. The excitation energy has been tuned between 2.4 and $2.65 \mathrm{eV}$. The samples studied emit PL under nonresonant excitation at energies around $2.43 \mathrm{eV}$ (3.5-ML well thickness) and $2.6 \mathrm{eV}$ (2.0-ML well thickness), with bandwidths of 55 and $32 \mathrm{meV}$, respectively.

Because the migration time depends on the amount of excess energy, ${ }^{32}$ in a first step we test the influence of migration processes on the dynamics by comparing the PL decay at a fixed detection energy for different excitation energies. Figure 6(a) shows the PL decay after excitation with excess energies of one and two LO-phonon energies. The decay is almost monoexponential, and shows the same dynamics, independent of the excess energy. This behavior is present for all detection energies within the PL band. Based on this result, we conclude that, after a fast relaxation within our time resolution, slow migration processes do not influence the dynamics, and that the decay is dominated by the lifetime of the localized excitons. Therefore, the lifetime of localized excitons can be studied as a function of quantum 
confinement when tuning the laser energy and thus exciting selectively localized excitons. Figure 6(b) shows the PL decay times for the 3.5 and the 2.0-ML sample determined directly from PL decay time after excitation one LO-phonon energy above the detection energy, which is the ordinate in Fig. 6(b).

For both samples, the PL decay time of the states decreases with increasing state energy, e.g., for the 2.0-ML sample from $280 \mathrm{ps}$ down to $60 \mathrm{ps}$. We attribute this change to an increase in radiative lifetime with increasing lateral confinement as the result of a decrease in the coherence volume. ${ }^{34}$ Nonradiative recombination should show the opposite behavior, since larger islands at lower energy have a higher probability to comprise a dislocation. Another possibility could be migration into lower states in energy, a process that is typically observed in disordered nanostructures, giving rise to an effective mobility edge. However, the independence of the decay time on the excitation energy, as discussed above, indicates that there is no significant migration into the states within the experimental time window. This implies that there is also no significant migration out of the states. The observed values of the lifetimes are in agreement with the expected change of the radiative lifetime due to lateral localization of excitons. The intrinsic lifetime of a free exciton in a II-VI quantum well is in the range of some picoseconds. In contrast, the band-to-band recombination time, which is the upper limit for the lateral confinement effect, is of the order of nanoseconds, and can be reached in the strong confinement limit. ${ }^{34,35}$ The measured values are in between these two extremes, as expected.

\section{HOMOGENEOUS LINEWIDTH OF LOCALIZED EXCITONS}

After discussing the dynamics on a picosecond time scale and thus dealing with the lifetime $T_{1}$, we now turn to the determination of the homogeneous polarization decay time $T_{2}$. By measuring $T_{2}$, the dephasing rate of localized exciton states can be determined as a function of localization depth. For example, in the ternary system of $\mathrm{CdS}_{x} \mathrm{Se}_{1-x}$ mixed crystals, the localization at alloy fluctuations results in an increase of $T_{2}$ due to the decreased density of final states for the scattering at low temperatures. ${ }^{36} \mathrm{~A}$ similar dependence is expected in the case of three-dimensional quantum confinement. We use an amplified Ti:Sa laser combined by an optical parametric amplifier to supply 80 -fs pulses tunable between $500 \mathrm{~nm}(2.48 \mathrm{eV})$ and $650 \mathrm{~nm}(1.9 \mathrm{eV})$ for a twobeam FWM experiment. The FWM signal is detected spectrally resolved, and time integrated in a reflection geometry of the direction of $2 \overrightarrow{k_{2}}-\overrightarrow{k_{1}}$ as a function of the delay time between the two incident beams with wave vectors $\overrightarrow{k_{1}}$ and $\vec{k}_{2}$. The central frequency of the excitation is tuned to excite resonantly the localized excitons in the 3.5-ML sample under investigation. The excitation intensity has been kept as low as possible in the range of $100 \mathrm{~nJ} / \mathrm{cm}^{2}$ per pulse.

Figure 7(a) shows the decay curves at different energies within the spectral response of the FWM signal. The decay of the FWM signal is almost monoexponential, and shows a delayed maximum and a fast quenching for negative delays, as expected for a strongly inhomogeneously broadened resonance. As can be seen in Fig. 7(a) the decay rate increases
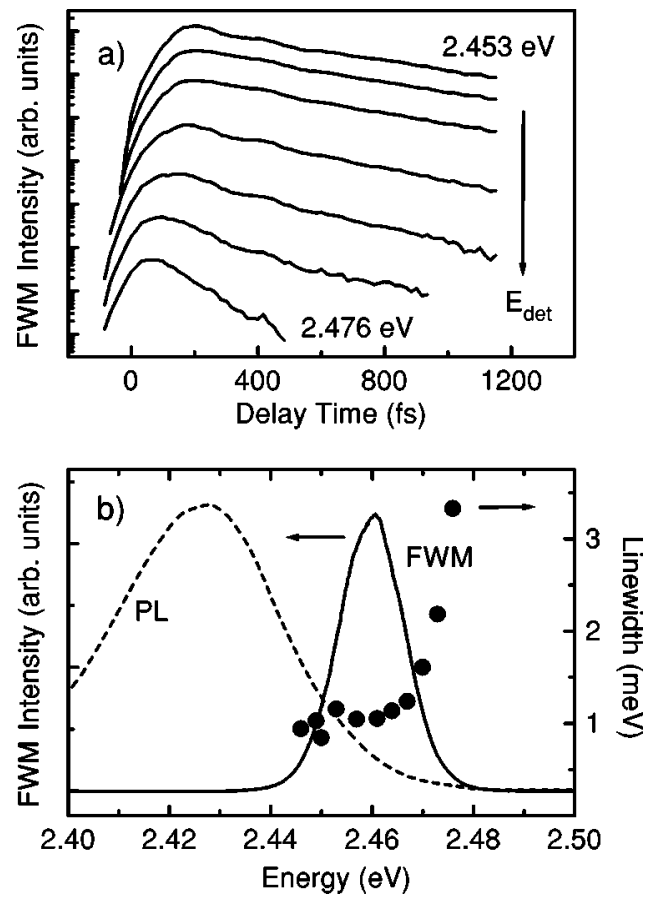

FIG. 7. a) Decay of the time-integrated FWM signal measured at different photon energies. (b) The PL spectrum (dashed line), the spectrally resolved FWM signal at zero delay (solid line), and the homogeneous linewidth $\Gamma$ (circles) are plotted as a function of the detection energy.

with increasing photon energy. This is evaluated in Fig. 7(b), where the PL spectrum, the spectrally resolved FWM signal, and the homogeneous linewidth $\Gamma$ as a function of detection energy (circles) are shown, using the relation $\Gamma=2 \hbar / T_{2} . T_{2}$ is four times the experimental decay time of the photon echo response from the inhomogeneously broadened system. Between 2.44 and $2.46 \mathrm{eV}$ the homogeneous linewidth is nearly constant and amounts to $\sim 1 \mathrm{meV}$. For detection at higher energies the linewidth increases, and reaches values up to 3.3 $\mathrm{meV}$. The observed increase in the homogeneous linewidth when probing higher energies is presumably due to an increasing phonon-assisted relaxation rate into lower localized exciton states, which becomes dominant in the high-energy wing of the PL band. For the structures presented here, the homogeneous linewidth $\Gamma$ is not influenced by effects of exciton-exciton interaction which might occur at higher excitation densities in experiments of nonlinear optics. Comparing $\Gamma$ derived from the FWM experiments with the homogeneous linewidth observed in low-density PL experiments at single excitons in etched mesa-structures, ${ }^{38}$ similar values of $\sim 1 \mathrm{meV}$ for the homogeneous linewidth are obtained. The values of $\Gamma$ reported here are distinctly different from the ultranarrow peaks in the range of $10-100 \mu \mathrm{eV}$ observed for localized excitons in III-V or II-VI nanostructures (see e.g., Refs. 12, 39, and 40). We attribute this increase in $\Gamma$ to a stronger coupling to acoustic phonons of the strongly confined excitons as theoretically predicted in Ref. 37.

\section{BIEXCITONS}

In this section we investigate the binding energies and the formation process of biexcitons in $\mathrm{Zn}_{1-x} \mathrm{Cd}_{x} \mathrm{Se} / \mathrm{ZnSe}$ quan- 


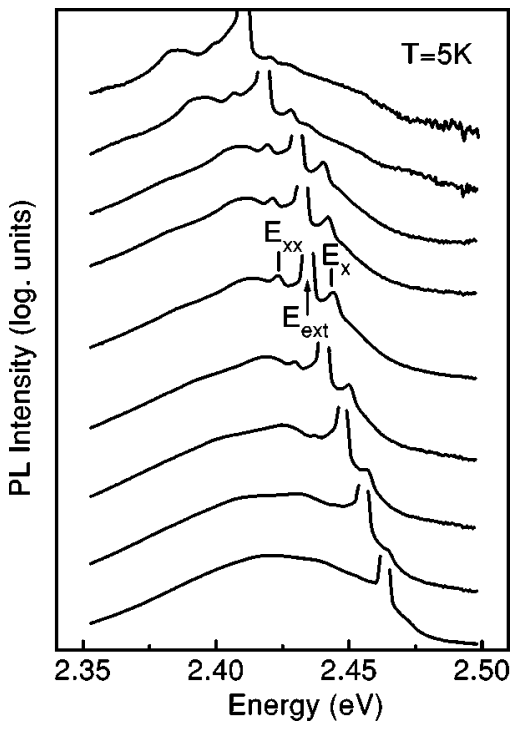

FIG. 8. Time-integrated PL spectra for different excitation energies $E_{\text {ext }}$ within the PL-band of localized exciton states. The resonantly excited biexciton-exciton and exciton-ground-state transitions are labeled $E_{\mathrm{xx}}$ and $E_{\mathrm{x}}$, respectively.

tum structures. We use two-photon absorption (TPA) and FWM spectroscopy to observe and identify the biexcitonic states. An increase in the biexciton binding energy compared to the bulk material is observed as the result of the threedimensional quantum confinement.

\section{A. Two-photon absorption}

Biexcitons can be created directly by resonantly enhanced two-photon absorption. Typically, in bulk semiconductors and quantum wells, this process is masked by a dominating one-photon exciton absorption due to the small binding energies of biexcitons. To observe the TPA into biexciton states, a negligible resonant one-photon absorption and a high excitation intensity are needed. TPA into localized biexciton states was reported for a single quantum well in Ref. 41. In the studies here, we use picosecond pulses of low repetition rate and excite resonantly in the tail of the density of states. Figure 8 shows the measured time-integrated PL spectra for different excitation energies within the PL band of localized exciton states. Besides a broad background due to exciton-exciton scattering and phonon-assisted relaxation, two peaks appear symmetrically around the energy of the exciting laser. These lines are not observed at low excitation densities. Tuning the excitation to higher energies corresponding to a higher density of states, one-photon absorption dominates, and the double structure disappears. In Fig. 9 the intensity dependence of the PL spectra is displayed at a fixed excitation energy of $2.434 \mathrm{eV}$. The peak intensities of the two symmetric lines, labeled $E_{\mathrm{x}}$ and $E_{\mathrm{xx}}$, grow superlinearly with respect to the first LO-phonon replica when increasing the excitation intensity. The energy separation between the excitation energy and the PL peaks is $\sim 10 \mathrm{meV}$. The peaks are suppressed for $\sigma^{+}$-polarized excitation of the same intensity (see Fig. 9). Both the intensity and the polarization dependencies indicate that the doublet is due to a resonantly enhanced two-photon absorption into a localized biexciton state. The corresponding level scheme in the inset of Fig. 9

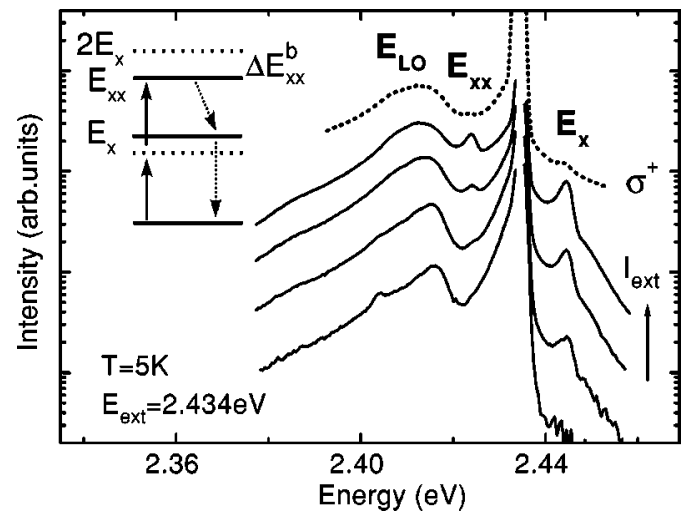

FIG. 9. Intensity dependence of the PL showing the resonantly excited exciton $\left(E_{x}\right)$ and the biexciton $\left(E_{x x}\right)$ transition and the LOphonon-assisted relaxation $\left(E_{\mathrm{LO}}\right)$. For comparison the spectrum is shown for excitation with circularly polarized light $\sigma^{+}$. The inset shows the scheme of transition energies for the exciton and the biexciton. $\Delta E_{x x}^{b}$ is the biexciton binding energy.

illustrates the excitation and recombination process of the biexcitons. The biexciton is directly excited by a resonantly enhanced TPA process, which consequently decays via the one-photon transitions from biexciton to exciton (low-energy peak $E_{x x}$ ), and exciton to ground state (high-energy peak $\left.E_{x}\right)$. In the investigated sample, the biexciton binding energy is $\sim 20 \mathrm{meV}$, given by the separation of the two side peaks $E_{x x}$ and $E_{x}$. For comparison, the bulk biexciton binding energy in CdSe is about $5 \mathrm{meV}$. $^{23}$

The dynamics of the two PL side bands confirm the recombination scheme. In Fig. 10 the PL spectra corresponding to the highest intensity of Fig. 9 are given for several delay times. Directly after excitation (10 ps), only $E_{x x}$ and $E_{\mathrm{LO}}$ are present, together with strong resonantly scattered laser, while the $E_{x}$ signal is absent, in agreement with its formation from the biexciton after the biexciton-exciton transition. At a 100-ps delay, both the $E_{x x}$ and the $E_{x}$ signals are present, showing the partial recombination of the biexciton into the exciton states. For a 300-ps delay time, the biexciton peak has vanished and only the exciton luminescence can be observed, due to the complete depopulation of the biexcitonic states. The measured decay time of the biexciton signal

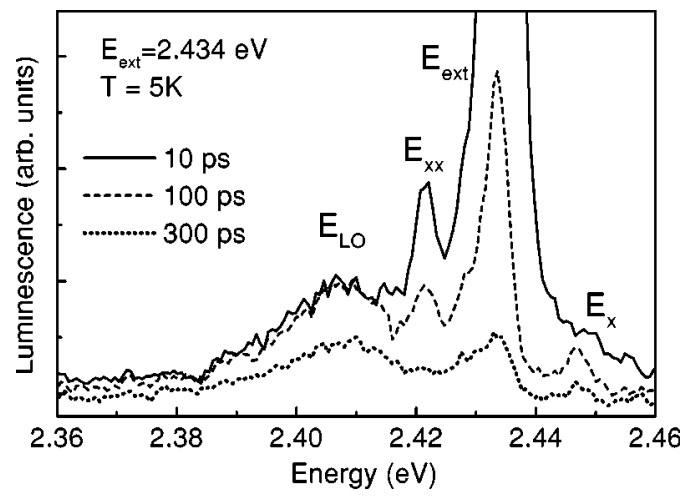

FIG. 10. Spectrally resolved PL at different delay times of 10 , 100 , and 300 ps. The spectral positions of exciton $E_{x}$, biexciton $E_{x x}$, the excitation $E_{\text {ext }}$ and the one LO-phonon replica $E_{\mathrm{LO}}$ are indicated. 

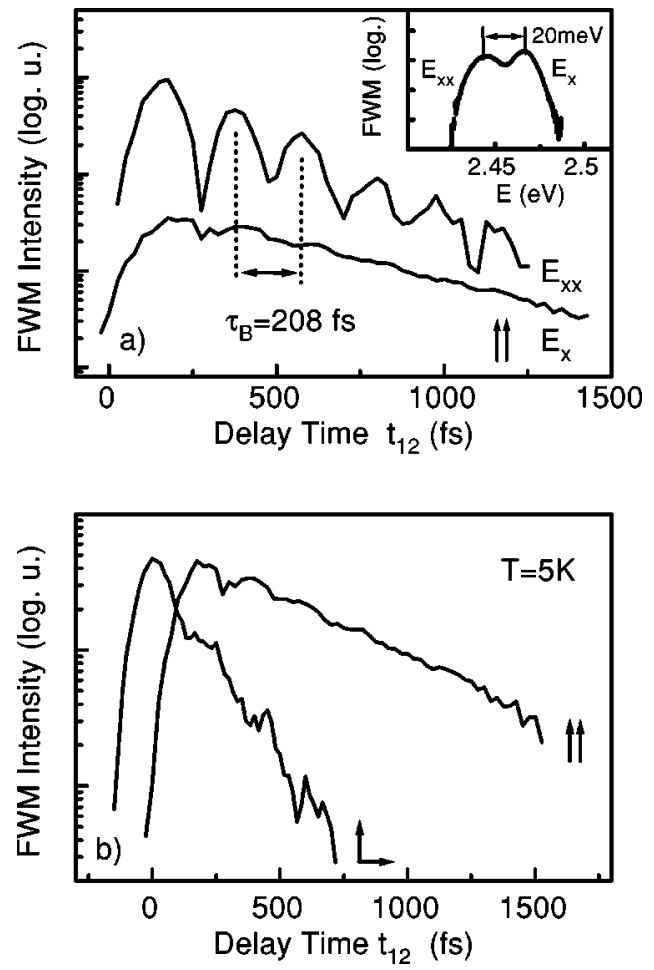

FIG. 11. (a) Time-integrated FWM signal from the 3.5-ML sample as function of delay time $t_{12}$ at an energetically position of $2.44 \mathrm{eV}\left(E_{x x}\right)$ and of $2.46 \mathrm{eV}\left(E_{x}\right)$. The two incident beams are colinearly polarized. The inset shows the spectral response at a delay time of $100 \mathrm{fs}$. (b) Time-integrated FWM signal detected at $\left(E_{x x}\right)$ for colinearly and cross-linearly polarized excitation. The experimental traces are normalized.

$E_{x x}$ of $60 \mathrm{ps}$ is in agreement with the rise of the $E_{x}$ exciton signal, which subsequently shows a decay time of twice the biexciton decay time, as expected.

\section{B. Four-wave mixing}

After analyzing the exciton-biexciton system in luminescence, we apply FWM to confirm the determined biexciton binding energy using coherent exciton-biexciton beats. The experimental setup is described in Sec. VI. The central frequency of the excitation is tuned into the localized excitons. The 80-fs pulses used ensure, by their large energy bandwidth, a simultaneous excitation of localized excitons and biexcitons. In Fig. 11(a) the time-integrated FWM signal of the 3.5-ML sample is shown as a function of the delay time $t_{12}$ at an energy of $2.44 \mathrm{eV}$, i.e., within an energy range for which the FWM response is strongly influenced by the biexcitonic signal. The signal reveals pronounced oscillations with a period of $208 \mathrm{fs}$ arising from the beating between the exciton and biexciton polarizations. At a higher detection energy of $2.46 \mathrm{eV}$, where the signal is dominated by the excitonic response, the beat amplitude is strongly reduced. The spectral response of the FWM-signal at 100-ps delay is shown in the inset. The beat period corresponds to an energy splitting of $20 \mathrm{meV}$. Good agreement between the oscillation period and the spectral separation of the exciton and biexciton in FWM and PL signals is obtained. Experimental evidence of the biexcitonic origin of the beats is also obtained from an analysis of the phase shift of the beat signals in case of different polarizations of the incident laser beams. According to Ref. 42, the biexcitonic beating in strongly inhomogeneously broadened systems starts with a minimum (maximum) at $t_{12}=0$ for colinear (cross-linear) polarization. In Fig. 11(b) the time-integrated FWM signal is shown for colinear and cross-linear polarized laser beams. In both configurations the signal is modulated, and the expected phase shift of $\pi$ can be observed between the two signals.

For the different samples a variation of the biexcitonic binding energy $\Delta E_{x x}^{b}$ between 20 and $24 \mathrm{meV}$ has been observed, depending on the strength of localization due to the composition fluctuations. The enhancement which the biexciton would experience in an ideal two-dimenaional (2D) quantum well can be estimated to $20 \%$ of the $2 \mathrm{D}$ exciton binding energy. ${ }^{43,44}$ Since the maximum $2 \mathrm{D}$ exciton energy is given by $4 E_{\mathrm{exc}}^{\text {bulk }}$ with $E_{\mathrm{exc}}^{\text {bulk }}=15 \mathrm{meV}$ (bulk exciton Rydberg of $\mathrm{CdSe}$ ), for the 2D biexciton binding energy an upper value around $12 \mathrm{meV}$ is expected. The experimentally determined biexciton binding energies clearly exceed that value. It is, however, in good agreement with theoretical and experimental results obtained for spherical CdSe quantum dots in glasses. ${ }^{45-48}$ Therefore, the measured large biexciton binding energies are a consequence of a strong lateral localization by the composition fluctuations, creating quantum-dot-like confined states. Since for $T=300 \mathrm{~K}$ thermal activation energies $\left(k_{B} T \sim 23 \mathrm{meV}\right)$ are still comparable to the biexciton binding energy, the high value of $\Delta E_{x x}^{b}$ makes biexcitons important even at room temperature.

\section{CONCLUSION}

As demonstrated by structural analysis and different methods of linear and nonlinear optics, migration enhanced epitaxy forces the accumulation of $\mathrm{CdSe}$ in islands embedded in a $\mathrm{Zn}_{1-x} \mathrm{Cd}_{x} \mathrm{Se}$ quantum well. The electronic states of such a system arise from a combined system of different dimensionalities, a strained quantum well, and a quantum dot. The observed gradient in the well composition favors the efficient carrier capture into the optically active islands and ensures high quantum efficiency. Concerning the application of the grown structures as a gain medium, two aspects have been revealed: (i) The large biexciton binding energy leads to an optical gain strongly influenced by Coulomb interaction similar to the biexcitonic gain observed in $\mathrm{CdSe}$ quantum dots embedded in glass. ${ }^{49,50}$ From our observation we conclude that strong optical pumping results in the formation of biexcitons rather than in a population and filling of higher excited dot states. As a consequence of the similar magnitude of biexciton binding energy and thermal energy $k_{B} T$, biexcitons are also important at room temperature, and an exact theoretical description of the gain mechanism has to take into account Coulomb correlation effects. (ii) The observed relaxation time into the ground state is very fast $(<4$ ps $)$ and supported by efficient phonon interaction. No competing emission is observed from the surrounding quantum well. These results are in agreement with the subpicosecond relaxation and gain dynamics observed for $\mathrm{CdSe}$ quantum dots embedded in glass. ${ }^{51,52}$ It implies that the car- 
rier distribution over the given density of states can reach thermal equilibrium on a picosecond time scale, allowing for high-speed devices. The analysis of the structural and optical properties of the quantum dot structures presented here demonstrated their attractive potential for applications in quantum dot laser devices emitting in the blue to green spectral range.

\section{ACKNOWLEDGMENTS}

The authors are grateful to S. Permogorov, C. Klingshirn, and F. Bechstedt for helpful discussions. We thank A. Rosenauer for support in the DALI analysis and T. Reimann in the FWM mixing experiments. The financial support of F.G. and K.L. by the DFG is gratefully acknowledged.
${ }^{1}$ F. Flack, N. Samarth, V. Nikitin, P. A. Crowell, J. Shi, J. Levy, and D. D. Awschalom, Phys. Rev. B 54, R17 312 (1996).

${ }^{2}$ S. H. Xin, P. D. Wang, Aie Yin, C. Kim, M. Dobrowolska, J. L. Merz, and J. K. Furdyna, Appl. Phys. Lett. 69, 3884 (1996).

${ }^{3}$ M. Löwisch, M. Rabe, B. Stegemann, F. Henneberger, M. Grundmann, V. Türck, and D. Bimberg, Phys. Rev. B 54, R11 074 (1996).

${ }^{4}$ Y. H. Wu, K. Arai, and T. Yao, Phys. Rev. B 53, R10 485 (1996).

${ }^{5}$ Hyun-Chui Ko, Doo-Cheol Park, Y. Kawakami, S. Fujita, and S. Fujita, Appl. Phys. Lett. 70, 3278 (1997).

${ }^{6}$ Z. Zhu, E. Kurtz, K. Arai, Y. F. Chen, D. M. Bagnall, P. Tomasini, F. Lu, T. Sekiguchi, T. Yao, T. Yasuda, and Y. Segawa, Phys. Status Solidi B 202, 827 (1997).

${ }^{7}$ K. Leonardi, H. Heinke, K. Ohkawa, D. Hommel, H. Selke, F. Gindele, and U. Woggon, Appl. Phys. Lett. 71, 1510 (1997).

${ }^{8}$ M. Strassburg, V. Kutzer, U. W. Pohl, A. Hoffmann, I. Broser, N. N. Ledentsov, D. Bimberg, A. Rosenauer, U. Fischer, and D. Gerthsen, Appl. Phys. Lett. 72, 942 (1998).

${ }^{9}$ M. Rabe, M. Lowisch, F. Kreller, and F. Henneberger, J. Comp. Neurol. 184/185, 248 (1998); H. Kirmse, R. Schneider, M. Rabe, W. Neumann, and F. Henneberger, Appl. Phys. Lett. 72, 1329 (1998).

${ }^{10}$ N. N. Ledentsov, I. L. Krestnikov, M. V. Maximov, S. V. Ivanov, S. L. Sorokin, P. S. Kopev, Zh. I. Alferov, D. Bimberg, and C. M. Sotomayor-Torres, Appl. Phys. Lett. 69, 1343 (1996).

${ }^{11}$ K. Leonardi, D. Hommel, C. Meyne, J.T. Zettler, and W. Richter, J. Cryst. Growth 201-202, 1222 (1999); K. Leonardi, H. Heinke, K. Ohkawa, D. Hommel, H. Selke, F. Gindele, and U. Woggon, J. Cryst. Growth 184/185, 259 (1998).

${ }^{12}$ V. Nikitin, P. A. Crowell, J. A. Gupta, D. D. Awschalom, F. Flack, and N. Samarth, Appl. Phys. Lett. 71, 1213 (1997).

${ }^{13}$ D. Hommel, K. Leonardi, H. Heinke, H. Selke, K. Ohkawa, F. Gindele, and U. Woggon, Phys. Status Solidi B 202, 835 (1997).

${ }^{14}$ M. Grün, F. Funfrock, P. Schunk, Th. Schimmel, M. Hetterich, and C. Klingshirn, Appl. Phys. Lett. 73, 1343 (1998).

${ }^{15}$ A. Rosenauer, T. Steinkirchner, J. Zweck, and W. Gebhardt, J. Cryst. Growth 152, 42 (1995).

${ }^{16}$ Z. Zhu, H. Yoshihara, K. Takebayashi, and T. Yao, Appl. Phys. Lett. 63, 1678 (1993).

${ }^{17}$ W. Shan, S. J. Hwang, J. M. Hayes, J. J. Song, Z. Q. Zhu, and T. Yao, J. Appl. Phys. 74, 5699 (1993).

${ }^{18}$ A. Rosenauer, S. Kaiser, T. Reisinger, J. Zweck, and W. Gebhardt, Optik (Stuttgart) 102, 63 (1996).

${ }^{19}$ U. Woggon, W. Langbein, J. M. Hvam, A. Rosenauer, T. Remmele, and D. Gerthsen, Appl. Phys. Lett. 71, 377 (1997).

${ }^{20}$ M. Grundmann, J. Christen, N. N. Ledentsov, J. Böhrer, D. Bimberg, S. S. Ruvimov, P. Werner, U. Richter, U. Gösele, J. Heidenreich, V. M. Ustinov, A. Yu. Egorov, A. E. Zhukov, P. S. Kopev, and Zh. I. Alferov, Phys. Rev. Lett. 74, 4043 (1995); M.
Grundmann, O. Stier, and D. Bimberg, Phys. Rev. B 52, 11969 (1995).

${ }^{21}$ S. Fafard, D. Leonard, J. L. Merz, and P. M. Petroff, Appl. Phys. Lett. 65, 1388 (1994).

${ }^{22}$ G. E. Pikus and G. L. Bir, Fiz. Tverd. Tela (Leningrad) 1, 1642 (1959) [ Sov. Phys. Solid State 1, 1502 (1959)].

${ }^{23}$ Semiconductors. Physics of II-VI and I-VII Compounds, Semimagnetic Semiconductors, edited by K.-H. Hellwege and O. Madelung, Landolt-Börnstein, New Series, Group III, Vol. 17, Pt. b (Springer-Verlag, Berlin, 1982).

${ }^{24}$ H. J. Lozykowski and V. K. Shastri, J. Appl. Phys. 69, 3235 (1991).

${ }^{25}$ A. G. Areshkin, G. S. Pekar', G. N. Polisskii, T. B. Popova, L. G. Suslina, and D. L. Fedorov, Fiz. Tverd. Tela (Leningrad) 28, 3743 (1986) [Sov. Phys. Solid State 28, 2109 (1986)].

${ }^{26}$ S. Permogorov, Phys. Status Solidi B 68, 9 (1975); S. Permogorov, A. Resnitzki, S. Verbin, and V. G. Lyssenko, Solid State Commun. 47, 5 (1983).

${ }^{27}$ F. Yang, P. J. Parbrook, B. Henderson, K. P. O’Donnell, P. J. Wright, and B. Cockayne, J. Lumin. 53, 427 (1992); F. Yang, B. Henderson, and K. P. O'Donnell, Solid State Commun. 88, 687 (1993).

${ }^{28}$ F. Gindele, U. Woggon, W. Langbein, J. M. Hvam, K. Leonardi, K. Ohkawa, and D. Hommel, J. Cryst. Growth 184/185, 306 (1998).

${ }^{29}$ M. Umlauff, J. Hoffmann, H. Kalt, W. Langbein, J. M. Hvam, M. Scholl, J. Söllner, M. Heuken, B. Jobst, and D. Hommel, Phys. Rev. B 57, 1390 (1998).

${ }^{30}$ M. Y. Valakh, A. P. Litvinchuk, R. S. Pekar, G. N. Polisskii, V. I. Sidorenko, and A. M. Yaremko, Phys. Status Solidi B 113, 635 (1982).

${ }^{31}$ U. Neukirch, D. Weckendrup, W. Faschinger, P. Juza, and H. Sitter, J. Cryst. Growth 138, 849 (1994).

${ }^{32}$ F. Yang, G. R. Hayes, R. T. Phillips, and K. P. O’Donnell, Phys. Rev. B 53, R1697 (1996).

${ }^{33}$ S. Yamaguchi, Y. Kawakami, S. Fujita, Sh. Fujita, Y. Yamada, T. Mishina, and Y. Masumoto, Phys. Rev. B 54, 2629 (1996).

${ }^{34}$ M. Sugawara, Phys. Rev. B 51, 10743 (1995).

${ }^{35}$ L. C. Andreani, in Confined Electrons and Photons: New Physics and Devices, edited by E. Burstein and C. Weisbuch (Plenum, New York, 1994), p. 1.

${ }^{36}$ H. Schwab, V. G. Lyssenko, J. M. Hvam, and C. Klingshirn, Phys. Rev. B 44, 3413 (1991).

${ }^{37}$ T. Takagahara, Phys. Rev. Lett. 71, 3577 (1993).

${ }^{38}$ F. Gindele, K. Hild, W. Langbein, and U. Woggon, Phys. Rev. B 60, R2157 (1999).

${ }^{39}$ D. Gammon, E. S. Snow, and D. S. Katzer, Appl. Phys. Lett. 67, 2391 (1995).

${ }^{40}$ T. Häupl, H. Nickolaus, F. Henneberger, and A. Schülzgen, Phys. Status Solidi B 194, 219 (1996). 
${ }^{41}$ K. Brunner, G. Abstreiter, G. Böhm, G. Tränkle, and G. Weimann, Phys. Rev. Lett. 73, 1138 (1994).

${ }^{42}$ T. F. Albrecht, K. Bott, T. Meier, M. Koch, S. T. Cundiff, J. Feldmann, W. Stolz, P. Thomas, S. W. Koch, and E. O. Göbel, Phys. Rev. B 54, 4436 (1996).

${ }^{43}$ W. Langbein and J. M. Hvam, Phys. Status Solidi B 206, 111 (1998).

${ }^{44}$ D. Birkedal, J. Singh, V. G. Lyssenko, J. Erland, and J. M. Hvam, Phys. Rev. Lett. 76, 672 (1996).

${ }^{45}$ Y. Z. Hu, M. Lindberg, and S. W. Koch, Phys. Rev. B 42, 1713 (1990).

${ }^{46}$ K. I. Kang, A. D. Kepner, S. V. Gaponenko, S. W. Koch, Y. Z. $\mathrm{Hu}$, and N. Peyghambarian, Phys. Rev. B 48, 15449 (1993).
${ }^{47}$ T. Takagahara, Phys. Rev. B 39, 10206 (1989).

${ }^{48}$ U. Woggon, O. Wind, W. Langbein, O. Gogolin, and C. Klingshirn, J. Lumin. 59, 135 (1994).

${ }^{49}$ U. Woggon, O. Wind, F. Gindele, E. Tsitsishvili, and M. Müller, J. Lumin. 70, 269 (1996).

${ }^{50}$ H. Giessen, U. Woggon, B. Fluegel, G. Mohs, Y. Z. Hu, S. W. Koch, and N. Peyghambarian, Chem. Phys. 210, 71 (1996); Opt. Lett. 21, 1043 (1996).

${ }^{51}$ H. Giessen, B. Fluegel, G. Mohs, N. Peyghambarian, U. Woggon, C. Klingshirn, P. Thomas, and S. W. Koch, J. Opt. Soc. Am. B 13, 1039 (1996).

${ }^{52}$ U. Woggon, H. Giessen, F. Gindele, O. Wind, B. Fluegel, and N. Peyghambarian, Phys. Rev. B 54, 17681 (1996). 